\title{
The diagnosis and management of infection following instrumented spinal fusion
}

\author{
Iona Collins · James Wilson-MacDonald • \\ George Chami - Will Burgoyne · P. Vineyakam • \\ Tony Berendt $\cdot$ Jeremy Fairbank
}

Received: 10 January 2007/Revised: 10 October 2007/Accepted: 18 November 2007/Published online: 13 December 2007

(c) Springer-Verlag 2007

\begin{abstract}
A 10-year retrospective audit. (1) The incidence of infection; (2) causative organisms; (3) whether eradication of infection is achievable with spinal implant retention; (4) patient outcome. The reported incidence of infection following posterior spinal instrumentation is between 2.6 and $3.8 \%$. Management of infection is controversial, with some advocating serial wound debridement while others report that infection cannot be eradicated with retention of implants. There are no published data demonstrating that propionibacteria are associated with early postoperative infection. The management of infected cases at our institution includes eventual removal of their implants. Our population was identified by studying the case notes of all patients who had undergone removal of spinal implants and cross-referencing this population with positive microbiology or histology reports. The incidence of infection was $3.7 \%$. Propionibacteria were isolated in $45 \%$ of cases. The diagnosis of infection was unexpected in $25 \%$ of patients, following removal of implants for prominence of implants or back pain. Sixty per cent of patients with acute postoperative deep wound infection had continuing active infection on subsequent removal of implants, despite longterm antibiotics and wound debridement. Fourty-six per cent of patients had a stable, pain-free spine at the end of their treatment. This is the largest reported series of infections following posterior spinal instrumented fusions
\end{abstract}

I. Collins · J. Wilson-MacDonald ( $₫)$ · G. Chami ·

P. Vineyakam · T. Berendt · J. Fairbank

Department of Spinal Surgery, Nuffield Orthopaedic Centre,

Windmill Rd, Headington, Oxford OX3 7LD, UK

e-mail: james.wilsonmacdonald@noc.anglox.nhs.uk

W. Burgoyne

Epsom, UK of which we are aware. Propionibacteria are a common cause of infection and successful eradication of infection cannot be reliably achieved with antibiotics and wound debridement alone.

Keywords Infection - Propionibacteria . Instrumentation · Diagnosis · Management

\section{Introduction}

Spinal infection following instrumented fusion is a difficult management problem. Accurate diagnosis is essential, in order to effectively eradicate the infecting organisms. Subsequent management of the diagnosed infection is controversial.

The reported incidence of infection following posterior spinal instrumentation is between $2.6 \%$ [9] and 3.8\% [12]. The incidence of delayed infection following posterior spinal instrumentation is between $0.2 \%$ [19] and 6.7\% [15].

Diagnosis of infection is difficult. Specific clinical signs, laboratory and radiographic investigations to aid in the diagnosis of infection may be absent; the diagnosis is often based on wound complications such as pain, inflammation and wound discharge.

In order to maximise the probability of isolating infecting organisms, we use a standardised tissue sampling protocol, as described by the Oxford Skeletal Infection Research and Intervention Service (OSIRIS) [1].

The objectives of this study were:

1. to report the incidence and nature of infection following posterior spinal instrumented surgery over a 10-year period;

2. to establish the nature of infecting organisms; 
3. to establish whether postoperative infection may be successfully eradicated by wound debridement and antibiotics alone and

4. to assess patient outcome.

\section{Materials and methods}

We reviewed the case notes of all patients diagnosed with deep wound infection following instrumented spinal fusion between 1993 and 2003. The routine management of infection following posterior spinal instrumented fusion at this institution includes the removal of implants where spinal fusion is established. Our population, therefore, was identified by studying the case notes of all patients who had undergone removal of spinal implants and cross-referencing this population with positive microbiology or histology reports.

Data collected from the infected cases are summarised in Table 1.

The diagnosis of infection was based on symptoms, such as persistent back pain, signs, such as wound fluctuance or discharge, raised inflammatory markers and radiographic features suggestive of infection. Infection at the time of implants removal was diagnosed when at least three of six samples sent to microbiology grew the same organism within 7 days. The microbiological results were correlated with histology findings to support the diagnosis. Our method of tissue sampling followed the OSIRIS collaboration protocol. A dedicated surgical tray with six separate sets of nibblers, forceps, scalpel handles and blades and specimen bottles was used to obtain eight tissue samples taken from areas immediately adjacent to the instrumentation, along the length of the wound. The location of each specimen relative to the metalwork was recorded at the time

Table 1 Data retrieved from case notes of infected instrumentation patients

\begin{tabular}{cl}
\hline Patient & Age \\
statistics & Gender \\
& Symptoms and signs indicating infection \\
& Residual symptoms following removal of metal ware \\
Procedure & Indication for surgery \\
& Spinal approach \\
& Type of prophylactic antibiotic cover \\
Infection & Organism \\
& Date infection diagnosed \\
& Date of metal ware removal \\
& Haematology, microbiology and histology \\
& investigations \\
& Types of antibiotics given to treat infection
\end{tabular}

of removal; each specimen was given a unique number from one to six. Six samples were sent for microbiology culture and microscopy and two were immediately fixed in formalin and sent for histological examination.

The management of infection following instrumented spinal fusion was undertaken in a dedicated Bone Infection Unit, under the care of the Infectious Diseases team. The treatment algorithm depended on whether the spine had fused at the time of diagnosis of infection. If unfused, each patient was given 6 weeks of intravenous antibiotics, then oral antibiotics until fusion was complete and the implants were then removed. In the fused spine, the management depended on the nature of the pathogen. Staphylococcus aureus and gram-negative bacterial infections were treated with 6 weeks intravenous antibiotics, then 6 weeks oral antibiotics with implant removal. Propionibacteria and coagulase-negative Staphylococcus bacteria were treated with 4 weeks of oral antibiotics in total, unless the host had a poor immune response, when the duration of treatment was extended with implant removal.

The assessment of patient outcome was made by recording symptoms and radiographic appearance of patients seen in clinic following removal of spinal instrumentation.

\section{Results}

Between 1993 and 2003, approximately 1980 instrumented spinal fusions were performed. During the same period, 74 infections were diagnosed. Fifty-three infections followed posterior spinal approaches and instrumented fusions, 21 infections followed anterior releases and posterior instrumented spinal fusions (all infections being in the posterior operation sites). There were no infections following anterior spinal instrumentation. This gave an infection incidence of $3.7 \%$.

All patients had been given a broad-spectrum prophylactic antibiotic at the index procedure, and latterly (after 2002) patients were given repeated doses of antibiotics two hourly where surgery times were more than $2 \mathrm{~h}$ while the wound remained open.

Almost all the implants during this period were made of stainless steel, although since 2003 we have used titanium implants exclusively.

There were 39 females and 35 males in this infected population. The median age was 24 years (range 11-77 years).

The indications for spinal instrumentation in this infected cohort are summarised in Table 2.

Sixteen infections were unexpected diagnoses following removal of implants for other indications, which were predominantly pain or prominent instrumentation. 
Table 2 Indication for instrumented spinal fusion

\begin{tabular}{lr}
\hline Trauma & 21 \\
Idiopathic scoliosis & 20 \\
Neuromuscular scoliosis & 9 \\
Spondylolisthesis & 8 \\
Kyphosis & 7 \\
Degenerative scoliosis & 4 \\
Discogenic back pain & 3 \\
Revision for non-union PLIF ${ }^{\text {a }}$ for trauma & 1 \\
Revision spinal decompression and PLIF & 1 \\
Total & 74 \\
\hline
\end{tabular}

${ }^{\text {a }}$ Posterolateral intervertebral fusion

The median time to diagnosis of infection was 14 months (7 days to 9 years postoperatively). Fifty-three infections were diagnosed within 2-year postoperatively (76\%), with six infections diagnosed within 30-day postoperatively (8\%). Eighteen were diagnosed after 2 years $(25 \%)$. Two patients had retention of implants pending fusion at the time of data collection. Fifty of the seventyfour infected cases had undergone multiple level fusions $(68 \%)$.

The incidence of deep infection following instrumented surgery for trauma was $5.3 \%$, following deformity correction was $6.7 \%$ and following degenerative spinal surgery, it was $1.1 \%$.

The median CRP was $37.5(<5-172)$, median ESR was 33.5 (2-100) and median WBC was 8.3 (3.3-12.3)

The infecting organisms are shown in Table 3. Fourtysix patients had single organisms isolated and 28 patients had polymicrobial infections. There was no correlation between patient age and presence of propionibacteria.

Figure 1 illustrates the length of time between the index procedure and the formal diagnosis of infection, as defined by positive microbiology and/or histology specimens.

Figure 2 illustrates the frequency and nature of organisms isolated following the index procedure. Figure 3

Table 3 Main bacteria isolated from intra-operative tissue samples

\begin{tabular}{lr}
\hline Propionibacteria & 34 \\
Staphylococcus & 32 \\
Coag neg staph & 18 \\
Staph aureus & 9 \\
MRSA & 5 \\
Proteus & 2 \\
Diphtheroids & 2 \\
E. coli & 2 \\
Anaerobic strep & 1 \\
Alpha haemolytic strep & 1 \\
\hline
\end{tabular}

Time between index surgery and samples for positive microbiology

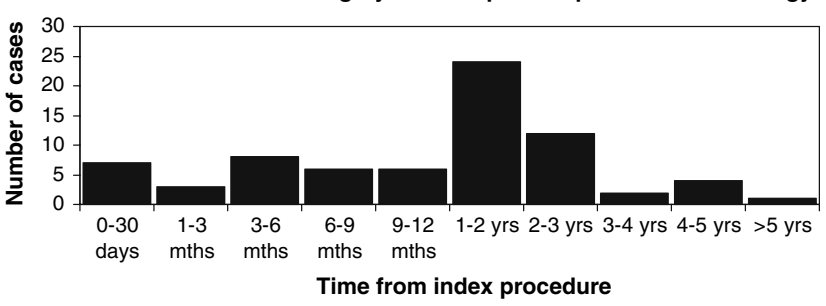

Fig. 1 Time between index surgery and samples for positive microbiology

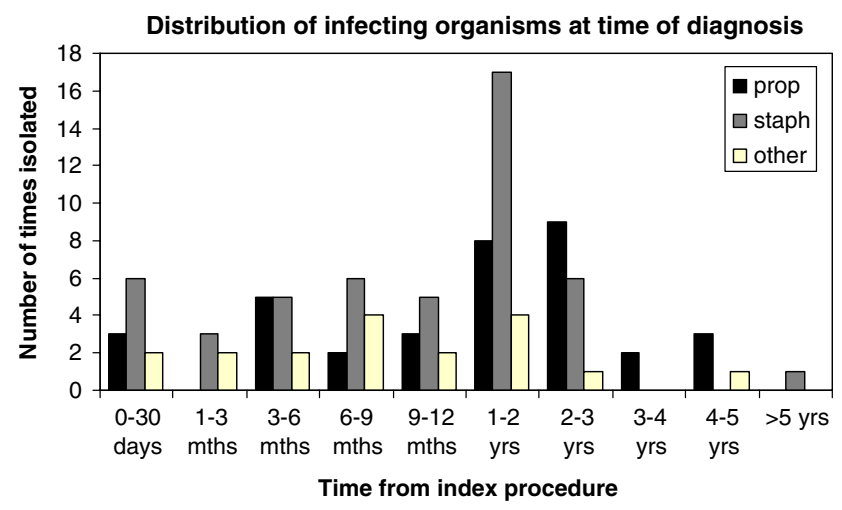

Fig. 2 Distribution of infecting organisms at time of diagnosis

illustrates the number of cases where propionibacteria were cultured in isolation.

Fifteen patients were treated with long-term antibiotics and wound debridement when indicated, prior to removal of their implants, as there was no established spinal fusion at the time of diagnosis of infection. Of these, nine had active infection at the time of their implants removal, four had no evidence of active infection and two patients had equivocal results, with positive histology for infection, but no growth on culture.

The median duration of intravenous antibiotic administration following implants removal was 4 weeks ( 4 days to

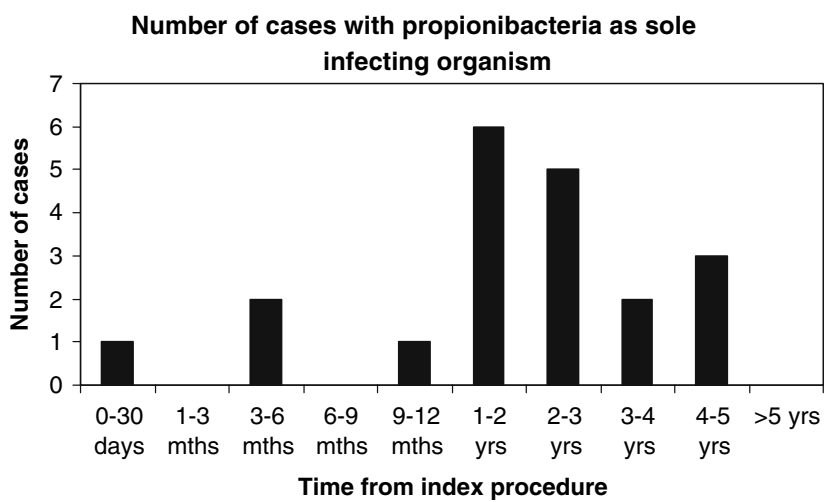

Fig. 3 Number of cases with propionibacteria as sole infecting organism 
Table 4 Symptoms and signs leading to diagnosis of infection/ removal of implants

\begin{tabular}{lr}
\hline Back pain & 16 \\
Open wound & 12 \\
Sinus & 6 \\
Metal ware protruding through skin & 3 \\
Prominent metal ware & 3 \\
Abscess & 2 \\
Fluctuant wound & 1 \\
Revision scoliosis correction & 1 \\
Unclear from notes & 30 \\
\hline
\end{tabular}

8 weeks) and the median duration of oral antibiotic administration was 5 weeks (nil given to 1 year and 6 months duration).

Table 4 summarises the symptoms and signs, where documented, which prompted either investigation for infection or removal of spinal implants for symptom relief. One infected case was diagnosed from intra-operative tissue samples sent during revision of posterior instrumented fusion for progressive scoliosis. The patient remained on long-term oral antibiotics, pending spinal fusion at the time of data collection. Of the 43 patients with documented signs prompting removal of implants, 19 patients $(44 \%)$ were diagnosed with spine infections based on intra-operative microbiology and histology tissue specimens sent on removal of spinal implants, either for back pain or prominence of implants. Data regarding integrity and bony purchase of the implants at the time of removal was available for 61 of the 74 patients. Fifty per cent of the implants were recorded as loose and $6 \%$ were broken at the time of implant removal.

Of the 74 patients, 16 patients had clearly documented pain-free stable spines (22\%) following treatment of their infections. Thirty-nine patients had no clear postoperative symptoms in their case notes at postoperative follow up visits. Excluding those patients without documented postoperative symptoms, 16 out of 35 , or $46 \%$ of patients had pain-free, stable spines following successful eradication of their infections. One patient underwent further instrumented surgery secondary to loss of spinal correction subsequent to removal of implants. There were no reported neurological complications resulting from our infection management regime.

Table 5 summarises patient symptoms, where recorded in the case notes.

\section{Discussion}

Our infection incidence of $3.7 \%$ is similar to the published literature for all indications. The infection rate for posterior
Table 5 Patient outcome following removal of implants

\begin{tabular}{lr}
\hline Asymptomatic & 16 \\
$\begin{array}{l}\text { Residual back pain } \\
\text { Persisting spine infection }\end{array}$ & $\begin{array}{l}\text { One sinus development in natal cleft } \\
\text { here sacral instrumentation left in } \\
\text { situ }\end{array}$ \\
$\begin{array}{lr}\text { Poor mobility and back pain } \\
\quad \text { as loss }\end{array}$ & 2 \\
$\quad$ of correction following r/o & \\
$\quad$ metal ware & 2 \\
Asymptomatic back, & \\
$\quad$ persisting infection & \\
elsewhere & \\
Improved back pain & \\
Painful pseudarthrosis & 39 \\
Insufficient data &
\end{tabular}

correction of neuromuscular deformity has been reported as high as $10 \%$ [14]. Twelve per cent of our infected cohort had neuromuscular scoliosis correction. Our rate of $3.7 \%$ includes those patients who had occult spinal infections, diagnosed only following removal of implants for indications other than overt infection. A quarter were detected more than 2 years following implantation. These figures suggest that even a 2 year postoperative follow-up period for documenting incidence of infection may underestimate the true incidence of postoperative infection.

Propionibacteria have been regarded by some as simply a culture contaminant [2] that should, therefore, be disregarded. Our data support existing reports demonstrating the pathogenicity of this organism; Richards and Hahn reported propionibacteria as a common cause of late-presenting spinal infection $[10,15]$. A recent retrospective series reported the presence of propionibaceria in 3\% of early infections (less than 30-day postoperatively) and $12 \%$ of late-presenting infections [11]. In our series, although all early infections cultured Staphylococcus species, 50\% of the early infections also isolated propionibacteria. The pathogenicity of propionibacteria at sites other than the spine has been well documented. Propionibacteria have caused 36 reported cases of endocarditis, three of which were fatal [5]. Propionibacteria are a recognised causes of ventriculo-peritoneal shunt infections and three cases of intracranial abscesses caused by the organism and successfully treated with penicillin have been reported [3].

We believe that our recorded detection of propionibacteria reflects meticulous sampling and culture methods that we undertook jointly with our microbiology department in Oxford, rather than tissue contamination. Propionibacteria are fastidious organisms that are facultative and in some cases, obligate anaerobes. In our hands, they typically require culture in cooked meat broth for 7 days for broths to become positive. Rather than our results representing 
contamination or a local problem, we suggest is it more likely that other centres are failing to diagnose these pathogens, and are attributing some late failures of implants requiring removal to mechanical problems, inflammation or culture-negative infection.

Seventeen per cent of CRP results, $45 \%$ of ESR and $95 \%$ of WBC results were within the normal range prior to the diagnosis of infection. These data reflect the unreliability of inflammatory markers as diagnostic markers of spinal implants infection when caused by low-grade pathogens.

Fifty-six patients were diagnosed with infection within 2 years of initial surgery $(75.7 \%)$ and 18 patients were diagnosed two or more years following initial surgery (24.3\%). One patient presented over 5 years following initial surgery. These data demonstrate the need for longterm follow-up following instrumented spinal surgery in order to obtain a more accurate incidence of infection.

Of the symptoms reported in patients' records, 24 presented with wound complications, 19 with back pain and 3 with prominent implants. Unfortunately, insufficient data were available in this study to calculate the percentage likelihood of infection associated with the presentation of back pain and prominent implants. There are no published data that provide a predictive outcome for infection associated with postoperative back pain. The prospective collection of these data would clarify the risk of infection in relation to isolated back pain and the likelihood of improved back pain with removal of implants.

Our data showed that only $46 \%$ of patients with documented postoperative symptoms had stable, pain-free spines at the end of their treatment, reflecting the high patient morbidity associated with infection following instrumented spinal fusion. This is in contrast to several reports of good outcomes following removal of lumbar instrumentation for low back pain [6,21]. Gaine et al. [8] reported on the outcome of six patients following removal of Isola implants for persistent low back pain, all of whom had compete resolution of their back pain following removal of instrumentation. The group performed thorough histological and microbiological analysis of intra-operative tissue samples during removal of the posterior instrumentation. One out of six cases had positive histology and microbiology for $S$. aureus infection and this patient was pain-free following removal of instrumentation.

The management of spinal implants infection is controversial, with some centres advocating serial wound washouts with retention of implants $[9,12,20]$, while others recommend removal of implants [15]. Improved infection-free survivorship has been reported using longterm oral suppressive antibiotic therapy as part of early infection management and instrumentation removal for late infection [11].
Theiss et al. [18] reported a significant incidence of loss of deformity correction and pseudarthrosis following removal of implants; they also pointed out that there was no evidence to suggest that spinal instrumentation inhibited the ability to treat postoperative spinal infection. Muschik et al. [13] also reported loss of correction following removal of implants and showed that one-stage revision of infected posterior instrumentation reduced this complication.

In our study, 41 patients had undergone correction of deformity with two patients subsequently losing spinal alignment following removal of their implants (4.9\%). One of these patients had a pseudarthrosis at the time of implants removal. Our experience would suggest that removal of metal in late cases of infection does not commonly result in the need for revision surgery to re-correct deformity. This finding differs with that of other centres, which have reported unacceptably high loss of correction following implant removal; Deckey et al. [7] found that 4 out of 14 patients (29\%) lost their sagittal correction following removal of long spinal arthrodesis, despite intraoperative confirmation of fusion at the time of implant removal. Longer follow up with larger numbers is required to establish the true risk of recurrence of significant deformity following removal of implants.

Of 15 patients in our study treated with antibiotic therapy \pm wound debridement and retention of implants, 9 (40\%) had active infection at the time of their implants removal despite their antibiotic therapy. This suggests that infection cannot always be eradicated with retention of implants. This finding is supported by previous reports which found that $21 \%$ of deep wound infections following neuromuscular scoliosis surgery had persistent wound discharge, which settled only after removal of implants [17]. Following removal of implants and subsequent antibiotic treatment, no patient in this series presented with recurrent infection, although one patient was still being treated for persistent infection at the time of data collection.

In conclusion, the commonest infecting organisms in our series were $S$. aureus and Propionibacteria. Propionibacteria were responsible for both early and late infections in our series. Propionibacteria is regarded as a low virulence organism and has only been reported as a late cause of postoperative infection following posterior instrumented spinal fusion $[4,15,16]$. This lack of data may reflect the difficulty in culturing these organisms, as they require enrichment cultures and prolonged incubation times (broths usually becoming cloudy at 6 or 7 days). The importance of adequate tissue sampling for both microbiology and histology cannot, therefore, be over-emphasised.

The incidence of infection following posterior instrumented spinal fusion is $3.7 \%$ in this study, with $24 \%$ of 
infections presenting at least 2 years postoperatively. A further $25 \%$ were occult infections, diagnosed serendipitously following removal of spinal implants. Isolated back pain may be the only clue to the existence of postoperative spinal infection. The percentage risk of infection associated with isolated back pain is unknown and may be the focus of future research.

The retention of implants when treating infection, even when symptoms and signs were successfully suppressed, was associated with the persistence of the infection, as identified by culture and histology of intra-operative tissue samples, in $60 \%$ of patients in our series. Retention of mechanically sound implants in early infection, to permit fusion to occur, is a legitimate strategy, but the need for subsequent removal of implants should be anticipated. Whether in this context or when implants is removed from an apparently fused spine at the time of presentation of late infection, removal of implants was associated with a low incidence of loss of deformity correction, providing there was no evidence of pseudarthrosis at the time.

Only $46 \%$ of patients with documented outcomes had stable, pain-free spines at the end of their treatment, reflecting the high patient morbidity associated with infection following instrumented spinal fusion. Given this, how best to prevent infection at the time of primary surgery is clearly an important topic for future research.

\section{References}

1. Atkins BL, Athanasou N, Deeks JL et al (1998) Prospective evaluation of criteria for microbiological diagnosis of prosthetic joint infection at revision arthroplasty. J Clin Microbiol 36(10):2932-2939

2. Ben-Galim P, Giladi M, Schwartz D, Ashkenazi E, Millgram M, Dekel S, Floman Y (2006) Association between sciatica and microbial infection: true infection or culture contamination? Spine 31(21):2507-2509

3. Chu R, Tummala R, Hall W (2001) Focal intracranial infections due to propionibacterium acnes: report of three cases. Neurosurgery 49(3):717-720

4. Clark C, Shufflebarger H (1999) Late-developing infection in instrumented idiopathic scoliosis. Spine 24(18):1909-1916

5. Clayton JJ, Baig W, Reynolds GW, Sandoe JAT (2006) Endocarditis caused by propionibacterium species: a report of three cases and a review of clinical features and diagnostic difficulties. J Med Microbiol 55:981-987
6. Cook S, Asher M, Lai S et al (2000) Reoperation after primary posterior instrumentation and fusion for idiopathic scoliosis: toward defining late operative site pain of unknown cause. Spine 25(4):463-468

7. Deckey JE, Court C, Bradford DS (2000) Loss of sagittal plane correction after removal of spinal implants. Spine 25(19):24532460

8. Gaine WJ, Andrew S, Chadwick P et al (2001) Late operative site pain with Isola posterior instrumentation requiring implant removal: infection or metal reaction? Spine 26(5):538-587

9. Glassman SD, Dimar JR, Puno RM et al (1996) Salvage of instrumented lumbar fusions complicated by surgical wound infection. Spine 21(18):2163-2169

10. Hahn F, Zbinden R, Min K (2005) Late implant infections caused by propionibacterium acnes in scoliosis surgery. Eur Spine $\mathrm{J}$ 14(8):783-788

11. Kowalski TJ, Berbari EF, Huddleston PM, Steckelberg JM, Mandrekar JN, Osmon DR (2007) The management and outcome of spinal implant infections: contemporary retrospective cohort study. Clin Infect Dis 44:913-20

12. Levi AD, Dickman CA, Sonntag VK (1997) Management of postoperative infection after spinal instrumentation. J Neurosurg 86:975-980

13. Muschik M, Luck W, Schlenzka D (2004) Implant removal for late-developing infection after instrumented posterior spinal fusion for scoliosis: re-instrumentation reduces loss of correction. A retrospective analysis of 45 cases. Eur Spine J 13(7):645-651

14. Perry JW, Montgomerie JZ, Swank S, Gilmore DS, Maeder K (1997) Wound infections following spinal fusion with posterior segmental spinal instrumentation. Clin Infect Dis 24:558-661

15. Richards BS (1995) Delayed infections following posterior spinal instrumentation for the treatment of idiopathic scoliosis. J Bone Joint Surg Am 77-A(4):524-529

16. Schofferman L, Zucherman J, Schofferman J et al (1991) Diptheroids and associated infections as a cause of failed instrument stabilization procedures in the lumbar spine. Spine 16(3):356358

17. Sponseller P, LaPorte DM, Hungerford MW et al (2000) Deep wound infections after neuromuscular scoliosis surgery: a multicenter study of risk factors and treatment outcomes. Spine 25(19):2461-2466

18. Theiss SM, Lonstein J, Winter R (1996) Wound infections in reconstructive spine surgery. Orthop Clin North Am 27(1):105110

19. Viola R, King H, Adler S et al (1997) Delayed infection after elective spinal instrumentation and fusion: a retrospective analysis of eight cases. Spine 22(20):2444-2450

20. Weinstein MA, McCabe JP, Cammisa FP (2000) Postoperative spinal wound infection: a review of 2,391 consecutive index procedures. J Spinal Disord Tech 13(5):422-426

21. Wild A, Pinto MR, Butler L et al (2003) Removal of lumbar instrumentation for the treatment of recurrent low back pain in the absence of pseudoarthrosis. Arch Orthop Trauma Surg 123(8):414-418 